\title{
Predictive ability of the ISS, NISS, and APACHE II score for SIRS and sepsis in polytrauma patients
}

\author{
Mica, L ; Furrer, E ; Keel, M ; Trentz, O
}

\begin{abstract}
Purpose: Systemic inflammatory response syndrome (SIRS) and sepsis as causes of multiple organ dysfunction syndrome (MODS) remain challenging to treat in polytrauma patients. In this study, the focus was set on widely used scoring systems to assess their diagnostic quality. Methods: A total of 512 patients (mean age: $39.2 \pm 16.2$, range: 16-88 years) who had an Injury Severity Score (ISS) 17 were included in this retrospective study. The patients were subdivided into four groups: no SIRS, slight SIRS, severe SIRS, and sepsis. The ISS, New Injury Severity Score (NISS), Acute Physiology and Chronic Health Evaluation II (APACHE II) scores, and prothrombin time were collected at admission. The Kruskal-Wallis test and 2-test, multinomial regression analysis, and kernel density estimates were performed. Receiver operating characteristic (ROC) analysis is reported as the area under the curve (AUC). Data were considered as significant if $\mathrm{p}<0.05$. Results: All variables were significantly different in all groups $(\mathrm{p}<0.001)$. The odds ratio increased with increasing SIRS severity for NISS (slight vs. no SIRS, 1.06, $\mathrm{p}=0.07$; severe vs. no SIRS, $1.07, \mathrm{p}=0.04$; and sepsis vs. no SIRS, $1.11, \mathrm{p}=0.0028$ ) and APACHE II score (slight vs. no SIRS, $0.97, \mathrm{p}=0.44$; severe vs. no SIRS, $1.08, \mathrm{p}=0.02$; and sepsis vs. no SIRS, 1.12, $\mathrm{p}=0.0028$ ). ROC analysis revealed that the NISS (slight vs. no SIRS, AUC 0.61; severe vs. no SIRS, AUC 0.67; and sepsis vs. no SIRS, AUC 0.77) and APACHE II score (slight vs. no SIRS, AUC 0.60; severe vs. no SIRS, AUC 0.74; and sepsis vs. no SIRS, AUC 0.82) had the best predictive ability for SIRS and sepsis. Conclusion: Quick assessment with the NISS or APACHE II score could preselect possible candidates for sepsis following polytrauma and provide guidance in trauma surgeons' decision-making.
\end{abstract}

DOI: https://doi.org/10.1007/s00068-012-0227-5

Posted at the Zurich Open Repository and Archive, University of Zurich

ZORA URL: https://doi.org/10.5167/uzh-71784

Journal Article

Published Version

Originally published at:

Mica, L; Furrer, E; Keel, M; Trentz, O (2012). Predictive ability of the ISS, NISS, and APACHE II score for SIRS and sepsis in polytrauma patients. European Journal of Trauma and Emergency Surgery, 38(6):665-671.

DOI: https://doi.org/10.1007/s00068-012-0227-5 


\title{
Predictive ability of the ISS, NISS, and APACHE II score for SIRS and sepsis in polytrauma patients
}

\author{
L. Mica $\cdot$ E. Furrer $\cdot$ M. Keel $\cdot$ O. Trentz
}

Received: 5 March 2012/ Accepted: 3 September 2012/Published online: 18 September 2012

(C) Springer-Verlag 2012

\begin{abstract}
Purpose Systemic inflammatory response syndrome (SIRS) and sepsis as causes of multiple organ dysfunction syndrome (MODS) remain challenging to treat in polytrauma patients. In this study, the focus was set on widely used scoring systems to assess their diagnostic quality.

Methods A total of 512 patients (mean age: $39.2 \pm 16.2$, range: $16-88$ years) who had an Injury Severity Score (ISS) $\geq 17$ were included in this retrospective study. The patients were subdivided into four groups: no SIRS, slight SIRS, severe SIRS, and sepsis. The ISS, New Injury Severity Score (NISS), Acute Physiology and Chronic Health Evaluation II (APACHE II) scores, and prothrombin time were collected at admission. The Kruskal-Wallis test and $\chi^{2}$-test, multinomial regression analysis, and kernel density estimates were performed. Receiver operating characteristic (ROC) analysis is reported as the area under the curve (AUC). Data were considered as significant if $p<0.05$.
\end{abstract}

\section{Mica $(\bowtie)$}

Division of Trauma Surgery, University Hospital of Zürich, 8091 Zurich, Switzerland

e-mail: ladislav.mica@usz.ch

E. Furrer

Division of Biostatistics, University of Zürich,

Zurich, Switzerland

M. Keel

University Hospital of Orthopedic Surgery, Inselspital Bern, Bern, Switzerland

O. Trentz

Department of Trauma Surgery, University Hospital of Zürich, Zurich, Switzerland
Results All variables were significantly different in all groups $(p<0.001)$. The odds ratio increased with increasing SIRS severity for NISS (slight vs. no SIRS, 1.06, $p=0.07$; severe vs. no SIRS, $1.07, p=0.04$; and sepsis vs. no SIRS, $1.11, p=0.0028$ ) and APACHE II score (slight vs. no SIRS, $0.97, p=0.44$; severe vs. no SIRS, 1.08 , $p=0.02$; and sepsis vs. no SIRS, $1.12, p=0.0028$ ). ROC analysis revealed that the NISS (slight vs. no SIRS, AUC 0.61 ; severe vs. no SIRS, AUC 0.67; and sepsis vs. no SIRS, AUC 0.77) and APACHE II score (slight vs. no SIRS, AUC 0.60 ; severe vs. no SIRS, AUC 0.74; and sepsis vs. no SIRS, AUC 0.82) had the best predictive ability for SIRS and sepsis.

Conclusion Quick assessment with the NISS or APACHE II score could preselect possible candidates for sepsis following polytrauma and provide guidance in trauma surgeons' decision-making.

Keywords SIRS · Sepsis · Polytrauma $\cdot$ ISS $\cdot$ NISS · APACHE II

\section{Introduction}

Trauma remains the main cause of death in urban environments among young adults and the middle-aged. Nowadays, traumatic injuries do not only lead to death directly by bleeding or destruction of pivotal organs, but also by the development of secondary diseases related to trauma, such as systemic inflammatory response syndrome (SIRS) and sepsis. The definition of SIRS outlined at the conference of the American College of Chest Physicians (ACCP) and the Society of Critical Care Medicine (SCCM) in 1992 is widely accepted [1]. Several publications indicate SIRS as an early warning sign of sepsis, especially in trauma patients with a 
large amount of destroyed soft tissue that can be indirectly quantified by the Injury Severity Score (ISS) and the New Injury Severity Score (NISS) [2-8]. In trauma patients, the main causes of immediate death are blood loss and the acute coagulopathy of trauma shock triggered at the trauma site [9]. Patients in shock are at high risk of acquiring infections at the injury site [10]. Although SIRS also promotes beneficial effects such as the clearance of pathogens and wound healing, the over activation of this mechanism may result in dysfunction of pivotal organs (multiple organ dysfunction syndrome [MODS]) [11, 12]. The compensatory antiinflammatory response syndrome (CARS) counteracts SIRS and, in combination with shock in trauma patients, can result in immunosuppression and increased susceptibility to infections [10, 11]. The acquisition of an infection may, again, enhance the physiological reaction of SIRS, and with an infectious focus, the patient fulfills the criteria for sepsis. Thus, the indirect quantification of soft tissue damage by the ISS and NISS may be insufficient to predict the possible development of SIRS or sepsis. The Acute Physiology and Chronic Health Evaluation II (APACHE II) score developed by Knaus et al. in the early 1980s summarizes the physiological state of the patient in the context of their chronic health status [13]. Laboratory parameters included in the APACHE II score quantify not only tissue damage, but also the ability of the body to handle trauma. Polytrauma patients are at high risk of developing acute coagulopathy of trauma shock [9]. Conventionally, acute coagulopathy of trauma shock is viewed as a later event mainly caused by resuscitative attempts occurring in the hospital, but there are patients admitted to the emergency department with an already established or evolving coagulopathy caused by trauma $[9,14-16]$. In these studies, there was a strong correlation between coagulopathy and mortality, and coagulopathy was identified as an independent risk factor [14].

The aim of this study was to determine the predictive ability for the development of SIRS and sepsis by widely used trauma scores such as the ISS and NISS, APACHE II score as a parameter that describes the physiological state at admission, and of prothrombin time as a mirror of acute coagulopathy of trauma shock as these parameters evolved as independent predictive parameters of death $[7,8,13,17]$.

\section{Materials and methods}

\section{Patients}

Five hundred and twelve polytrauma patients admitted to the emergency room of the University Hospital of Zürich in the period 1996-2002 were included in this study. Admission criteria were an ISS $\geq 17$ points, age $\geq 16$ years, and an admission time within $24 \mathrm{~h}$ of suffering a polytrauma
(ISS $\geq 17$ ). The patients were subjected to intensive care treatment and damage-control surgery. The population was subdivided into four groups: patients who did not fulfill any SIRS criteria, patients who fulfilled at least two SIRS criteria, patients who fulfilled three or four SIRS criteria at the day of admission, and patients who fulfilled the criteria for sepsis at any time during their hospitalization. All patient data were collected retrospectively according to the guidelines of "Good Clinical Practice". The ISS, NISS, and APACHE II scores were defined based on the data collected at admission to the emergency department during the first $24 \mathrm{~h}$. Patients primarily treated in other hospitals and patients who died within the first 3 days were excluded. All data were retrieved from patient records as approved by the local institutional review board (IRB) according to the University of Zürich IRB guidelines and the study was conducted according to the guidelines of good clinical practice ("Retrospektive Analysen in der Chirurgischen Intensivmedizin" Nr. StV. 01-2008).

\section{Surgical treatment}

The treatment of all admitted patients followed the Advanced Trauma Life Support (ATLS $®$ ) guidelines and the previously assessed trauma management protocol [18-20]. Briefly, after airway intubation, ventilation, and cardiovascular management, life-saving surgery took place with decompression of body cavities, control of hemorrhage, and identification of contaminated tissue. The first surgical interventions were followed by stabilization of major fractures and radical debridement of dead tissue. Cefazolin was used as the perioperative antibiotic. In all of the admitted patients, enteral nutrition was established within $24 \mathrm{~h}$ after trauma to avoid spontaneous transmigration of the enteric microbial flora and peritoneal contamination.

\section{Trauma-scoring systems}

The ISS and NISS were used to define the severity of trauma $[7,8]$. The APACHE II score was used to evaluate the overall physiological impairment of the patient [13].

Prothrombin time measurement

The prothrombin time was measured at admission of the patient by a standardized, previously described method [16].

Statistical analysis

Continuous data are presented as mean \pm standard deviation (SD) and are compared between groups using the Kruskal-Wallis test. Descriptive statistics include frequencies and percentages for categorical data and the 
$\chi^{2}$-test was used for proportions. The diagnostic quality of continuous variables was assessed using receiver operating characteristic (ROC) curves and the area under the curve (AUC). For the AUC, Wald confidence intervals were computed on the logit scale and retransformed. Proportional odds models were fitted in order to assess the predictive ability of the parameters for the development of SIRS and sepsis. To obtain density estimates for the score values, a log-concavity assumption was used [21]. This is a nonparametric method to estimate density that does not suffer from the potential deficiencies of kernel density estimates, such as the need to choose the kernel, bandwidth, and wiggles. Comparison of our density estimates with histograms revealed that the log-concavity assumption was accurate. To describe further the score distributions, mode estimates computed from the above densities were provided. The mode is the value on the $x$-axis where the estimated density reaches its maximum. All computations were done in $\mathrm{R}$ [22]. log-concave density estimates were computed using the $\mathrm{R}$ package logcondens [23]. The statistical analysis was performed by the Institute for Biostatistics of the University of Zürich.

\section{Results}

Patient sample

A total of 512 patients fulfilling the criteria for polytrauma (ISS $\geq 17$ ) and with an average age of $39.1 \pm 16.6$ years (range: 16-88 years) were included in this study; 393 $(76.8 \%)$ were male and $119(23.3 \%)$ were female. A total of 169 males ( $43 \%$ of males) developed severe SIRS, compared with 47 females (39.5\% of females) $(p<0.001)$ (Table 1). Sepsis was observed in 97 males $(24.7 \%$ of males) and in 20 females $(16.8 \%$ of females $)(p<0.001)$ (Table 1). In total, 59 patients died: in the no SIRS group, all patients survived; in the slight SIRS group, seven patients died; in the severe SIRS group, 28 patients died; and in the sepsis group, 24 patients died. The main cause of death was either a brain/skull injury or MODS (Table 2).

Predictive ability of the polytrauma scores for SIRS and sepsis

First, the diagnostic quality of the polytrauma scores and of prothrombin time have been assessed by the AUC, showing that the APACHE II score has the best diagnostic quality, followed by the NISS, prothrombin time, and the ISS (Table 3). The proportional odds model allows investigators to derive predictive probabilities for the severity of SIRS and sepsis given increasing values of the polytrauma scores. The data show that all parameters have a significant predictive effect for SIRS and sepsis (Table 4). Proportional odds imply that, for example, for a one unit increase in the APACHE II score, the odds of developing severe SIRS or sepsis are $10 \%$ higher than of developing no SIRS or only slight SIRS.

The model predicts that increasing NISS and APACHE II score implies, on the one hand, that the predictive

Table 1 Characteristics of the patient sample

\begin{tabular}{|c|c|c|c|c|c|}
\hline Characteristics & No SIRS & Slight SIRS & Severe SIRS & Sepsis & $p$-value \\
\hline Number $[N](\%$ of all $)$ & $75(14.6)$ & $104(20.3)$ & $216(42.2)$ & $117(22.9)$ & \\
\hline Age [years $\pm \mathrm{SD}$ ] & $41.4 \pm 18.0$ & $38.8 \pm 16.0$ & $38.6 \pm 17.2$ & $38.6 \pm 15.2$ & $0.693^{\dagger}$ \\
\hline Males $[N]$ (\% of males) & $52(13.2)$ & $75(19.1)$ & $169(43.0)$ & $97(24.7)$ & $<0.001^{*}$ \\
\hline Females $[N]$ (\% of females) & $23(19.3)$ & $29(24.4)$ & $47(39.5)$ & $20(16.8)$ & $<0.001^{*}$ \\
\hline Male/female [\% of each subgroup] & $69.3 / 30.7$ & $72.1 / 27.9$ & $78.2 / 21.8$ & $82.9 / 17.1$ & $<0.05^{\star}$ \\
\hline ISS [points $\pm \mathrm{SD}$ ] $(95 \% \mathrm{CI})$ & $\begin{array}{l}27.5 \pm 8.3 \\
(25.6,29.4)\end{array}$ & $\begin{array}{l}30.2 \pm 9.9 \\
\quad(28.3,32.2)\end{array}$ & $\begin{array}{r}33.0 \pm 12.4 \\
(31.3,34.7)\end{array}$ & $\begin{array}{r}37.3 \pm 12.9 \\
(34.9,39.6)\end{array}$ & $<0.001^{\dagger}$ \\
\hline NISS [points $\pm \mathrm{SD}$ ] $(95 \% \mathrm{CI})$ & $\begin{array}{r}34.1 \pm 10.4 \\
(31.7,36.5)\end{array}$ & $\begin{array}{r}38.5 \pm 11.9 \\
(35.7,40.6)\end{array}$ & $\begin{array}{l}42.0 \pm 14.0 \\
(42.0,43.9)\end{array}$ & $\begin{array}{r}46.5 \pm 12.9 \\
(44.2,48.9)\end{array}$ & $<0.001^{\dagger}$ \\
\hline $\begin{array}{l}\text { APACHE II [points } \pm \text { SD] } \\
(95 \% \mathrm{CI})\end{array}$ & $\begin{array}{r}10.2 \pm 6.8 \\
(8.6,11.7)\end{array}$ & $\begin{array}{l}12.4 \pm 7.2 \\
(11.0,13.8)\end{array}$ & $\begin{array}{r}16.4 \pm 7.9 \\
(15.4,17.5)\end{array}$ & $\begin{array}{r}18.7 \pm 6.9 \\
(17.4,19.9)\end{array}$ & $<0.001^{\dagger}$ \\
\hline $\begin{array}{l}\text { Prothrombin time }[\% \pm \mathrm{SD}] \\
\quad(95 \% \mathrm{CI})\end{array}$ & $\begin{array}{c}89.8 \pm 14.9 \\
(77.7,90.0)\end{array}$ & $\begin{array}{l}86.1 \pm 16.3 \\
\quad(77.2,86.7)\end{array}$ & $\begin{array}{r}78.5 \pm 19.5 \\
(72.3,78.8)\end{array}$ & $\begin{array}{r}73.5 \pm 20.3 \\
(67.3,75.8)\end{array}$ & $<0.001^{\dagger}$ \\
\hline
\end{tabular}

SIRS systemic inflammatory response syndrome, ISS Injury Severity Score, NISS New Injury Severity Score, APACHE II Acute Physiology and Chronic Health Evaluation II

Data are given as mean \pm standard deviation (SD), with $95 \%$ confidence interval $(\mathrm{CI})$ in parentheses

$* \chi^{2}$-test

$\dagger$ Kruskal-Wallis test

* Mann-Whitney test 
Table 2 Characteristics and cause of death of participants

\begin{tabular}{|c|c|c|c|c|}
\hline Death & No SIRS & Slight SIRS & Severe SIRS & Sepsis \\
\hline Number $[N](\%$ of all $)$ & $0(0)$ & $7(1.4)$ & $28(5.5)$ & $24(4.7)$ \\
\hline Males $[N]$ (\% of males) & $0(0)$ & $6(1.5)^{*}$ & $25(6.3)^{*}$ & $24(6.1)^{*}$ \\
\hline Females $[N]$ (\% of females) & $0(0)$ & $1(0.8)$ & $3(1.8)$ & $0(0)$ \\
\hline Cause of death: multiorgan failure & 0 & 1 & 9 & 22 \\
\hline Cause of death: brain/skull injury & 0 & 6 & 19 & 2 \\
\hline
\end{tabular}

An increasing severity of systemic inflammatory response syndrome (SIRS) leads to an increased death rate in both males and females. In this patient sample, no sepsis was observed in females. The death rate due to SIRS and sepsis is significantly higher in the male sample $\left(* \chi^{2}\right.$-test, male vs. female, $p<0.05$ ). The main cause of death in the SIRS group was brain or skull injury and in the sepsis group, it was multiple organ dysfunction syndrome (MODS)

Table 3 Coefficients for the outcomes of each pair of SIRS status groups derived by the multimodal logistic regression model

\begin{tabular}{llll}
\hline & NISS & $\begin{array}{l}\text { APACHE II } \\
\text { score }\end{array}$ & $\begin{array}{l}\text { Prothrombin } \\
\text { time }\end{array}$ \\
\hline $\begin{array}{l}\text { Slight versus no SIRS } \\
\quad\end{array}$ & 1.06 & 0.97 & 0.97 \\
$\quad \begin{array}{l}\text { Odds ratio } \\
p \text {-value }\end{array}$ & 0.07 & 0.44 & 0.11 \\
95\% CI for odds ratio & 0.99, & $0.90,1.05$ & $0.94,1.01$ \\
& 1.14 & & \\
Severe versus no SIRS & & & 0.97 \\
Odds ratio & 1.07 & 1.08 & 0.06 \\
$p$-value & $\mathbf{0 . 0 4}$ & $\mathbf{0 . 0 2}$ & $0.94,1.00$ \\
C95\% CI for odds & 1.00, & $1.01,1.16$ & \\
ratio & 1.14 & & 0.97 \\
Sepsis versus no SIRS & & & 0.07 \\
Odds ratio & 1.11 & 1.12 & $0.94,1.00$ \\
$p$-value & $\mathbf{0 . 0 0 2 8}$ & $\mathbf{0 . 0 0 2 8}$ & \\
95\% CI for odds ratio & 1.04, & $1.04,1.21$ & \\
& 1.19 & &
\end{tabular}

For the NISS and APACHE II score, the level of significance increases with more severe disease, and the predictive quality of the NISS and APACHE II score increases with increasing severity of SIRS toward sepsis (bold)

CI confidence interval

probability of contracting no or only slight SIRS decreases and, on the other hand, that the probability of contracting severe SIRS or even sepsis increases (Table 4). For example, a value of 9 for the APACHE II score (corresponding to the 25 th percentile) entails probabilities of $20 \%$ for no SIRS, $27 \%$ for slight SIRS, $41 \%$ for severe SIRS, and $13 \%$ for sepsis. For a value of 20 for the APACHE II score (corresponding to the 75th percentile), the first two probabilities are decreased to 8 and $16 \%$ and the second two probabilities are increased to 47 and $29 \%$.

Density estimates of the ISS, NISS, APACHE II score, and prothrombin time

In order to provide 'threshold values' for each SIRS category to show possible orientation points, densities have
Table 4 Shown is the predictive quality as the area of the curve (AUC) of the receiver operating characteristic (ROC) curve and $95 \%$ $\mathrm{CI}$ in parentheses

\begin{tabular}{llll}
\hline $\begin{array}{l}\text { No SIRS } \\
\text { vs. }\end{array}$ & NISS & $\begin{array}{l}\text { APACHE II } \\
\text { score }\end{array}$ & $\begin{array}{l}\text { Prothrombin } \\
\text { time }\end{array}$ \\
\hline $\begin{array}{c}\text { Slight } \\
\text { SIRS }\end{array}$ & $0.61(0.49,0.72)$ & $0.60(0.49,0.69)$ & $0.58(0.42,0.72)$ \\
$\begin{array}{c}\text { Severe } \\
\text { SIRS }\end{array}$ & $0.67(0.56,0.76)$ & $0.74(0.65,0.81)$ & $0.68(0.54,0.79)$ \\
Sepsis & $0.77(0.66,0.86)$ & $0.82(0.73,0.88)$ & $0.74(0.60,0.84)$ \\
\hline
\end{tabular}

Increasing severity of SIRS and sepsis gives better predictive quality for each parameter except prothrombin time, where decreasing values give better predictive quality

been estimated using a log-concavity assumption [23]. From the density estimates, the modes for no SIRS, slight SIRS, severe SIRS, and sepsis were estimated for the ISS (mode: no SIRS 22.9, slight SIRS 25, severe SIRS 25.5, and sepsis 34.5 points), NISS (mode: no SIRS 31, slight SIRS 33.3, severe SIRS 31.9, and sepsis 46.4 points), APACHE II score (mode: no SIRS 6.3, slight SIRS 7.6, severe SIRS 17.8, and sepsis 18.7 points), and prothrombin time (mode: no SIRS 94.2\%, slight SIRS 91.8\%, severe SIRS $90.0 \%$, and sepsis $87.2 \%$ ) (Fig. 1). These density estimates show the cumulative peaks for all patients included in each SIRS/sepsis group.

\section{Discussion}

The concept of SIRS has been evolving since its description in 1992 [1]. The sequence of SIRS to sepsis and septic shock has been used to predict the outcome in hospitalized patients [1, 24-26]. Data analyses of widely used traumascoring systems in the context of SIRS and sepsis are scarce. The incidence of SIRS in polytrauma patients is high, but not every polytrauma patient develops clinical signs of SIRS. In this study, we focused on widely used scoring systems such as the ISS, NISS, and APACHE II score and on prothrombin time as a mirror of acute 
Fig. 1 Density estimates of the Injury Severity Score (ISS), New Injury Severity Score (NISS), Acute Physiology and Chronic Health Evaluation II (APACHE II) score, and prothrombin time in graphical and numerical form. The scores increase with the severity of systemic inflammatory response syndrome (SIRS), except prothrombin time, which decreases with the severity of SIRS and sepsis
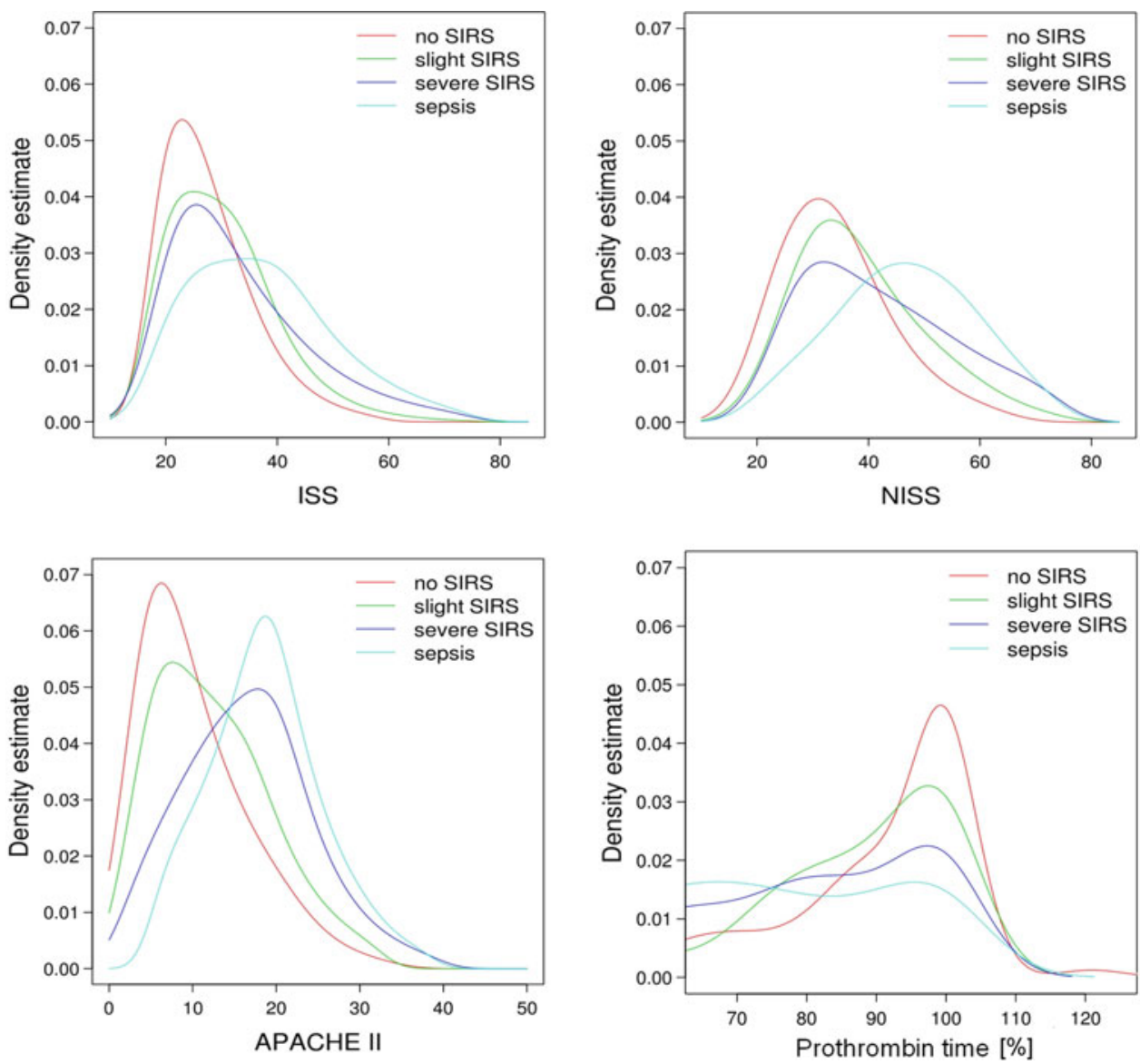

\begin{tabular}{l|c|c|c|c} 
& ISS & NISS & APACHE II & Prothrombin time \\
\hline No SIRS & 22.9 & 31 & 6.3 & $99.1 \%$ \\
\hline Slight SIRS & 25 & 33.3 & 7.6 & $97.5 \%$ \\
\hline Severe SIRS & 25.5 & 31.9 & 17.8 & $97.4 \%$ \\
\hline Sepsis & 34.5 & 46.4 & 18.7 & $67.3 \%$
\end{tabular}

coagulopathy related to trauma shock, and assessed their predictive ability for SIRS or sepsis [9]. Here, the values of these widely used trauma-scoring systems and their predictive quality for SIRS should be described.

Both the ISS and NISS add values according to the severity of injury of each anatomical region; the scores of the three most severe injuries are squared and summed. The sum of the three squared numbers represents the score. The main difference between the ISS and NISS is that the NISS can count multiple injuries in the same anatomical region. This difference does not change the predictive ability of the NISS for SIRS and sepsis (Table 4.). The data show that the NISS has a higher predictive quality for severe SIRS and sepsis than the ISS (Table 3). The reason for this difference remains unclear, and may be a statistical anomaly. Both scores describe the severity of injury in a certain anatomical region and describe tissue damage. The slight difference between the scoring rules of the ISS and NISS may be the reason for the slight difference in their ability to predict SIRS and sepsis. In the NISS, the three most severe injuries that are counted may lie in the same anatomical region, which, hypothetically, may give a higher value for effective tissue damage than in the ISS. It is well known that, upon cellular damage, hidden antigens (damage-associated molecular patterns [DAMPs]), such as uric acid and hyaluronic acid fragments, are released, initiating nonspecific immunological reactions [27]. Depending on the concentration of DAMPs, the immunological reaction may lead to SIRS and, when an infection is present, to sepsis; i.e., the more DAMPs, the greater the risk of developing signs of SIRS.

The high predictive quality of the APACHE II score for the development of SIRS or sepsis is partially explained by the fact that the SIRS criteria are included in the APACHE 
II score. However, the predictive quality should, therefore, be higher than 0.82 AUC. The reason for this lower than expected predictive quality may lie in the complexity of the APACHE II score, which also takes account of the patient's chronic health status. The APACHE II score for each patient collected at admission reflects the immediate physiological status relative to their chronic health status, so unhealthy and older patients have a higher APACHE II score and probably develop SIRS or sepsis more easily because of a lower compensatory ability. Resuscitative procedures carried out according to the $\operatorname{ATLS}^{\circledR}$ criteria may influence the hematocrit and electrolyte balance, especially in bleeding patients, and increase the APACHE II score. The degree to which the APACHE II score of a polytrauma patient at admission is iatrogenic remains speculative.

The overall odds ratio of the prothrombin time was 1.03 (Table 4), indicating the lowest predictive quality for SIRS and sepsis. The role of this parameter may be more indirect: a lower prothrombin time leads to increased blood loss and, thus, to an increased APACHE II score. Blood loss itself is a direct risk factor for the acquisition of infections [10]. The data presented here indicate that the prothrombin time alone cannot be used as a predictor for SIRS and sepsis, but can be used as an indirect indicator of further complications in polytrauma patients.

Female sex seems to have a protective effect against sepsis after polytrauma (Table 2) [28]. Interestingly, female subjects also developed SIRS significantly less frequently than males, probably because of the protective effect of 17- $\beta$-estradiol [28]. The question still remains as to how we can measure SIRS or sepsis. The complementary system may provide not only a therapeutic but also a quantifying opportunity to measure inflammatory reaction in patients. C5a is an inflammatory peptide with a broad spectrum of biological functions and is elevated during inflammatory reactions. In a murine sepsis model, cecal ligation and puncture and the inhibition of C5a led to an increased survival rate [29]. Measuring proinflammatory components of the complementary system may provide a quantification method of SIRS and sepsis.

The present study may provide guidance for estimating the risk of polytrauma patients developing SIRS or sepsis. The anticipation of septic problems could lead to quicker decisions in intensive care unit conditions in polytrauma patients and, therefore, improve the outcome for such patients and reduce their overall mortality.

\section{Limitations of the study}

Seemingly, the main limitation of this study is the analysis of the values at admission without taking into account surgical interventions. However, each surgical intervention such as debridement should only perioperatively increase the symptoms of SIRS. Normally, the patients do better after surgery. To avoid treatment bias, the data were collected at admission of the patients within the first $24 \mathrm{~h}$ or before the first surgical intervention. The course of SIRS is very dynamical and may change very quickly; therefore, the APACHE II score evaluated within the first $24 \mathrm{~h}$ was taken in order to avoid surgical treatment bias.

Conflict of interest There are no conflicts of interest among the authors.

\section{References}

1. Bone RC, Balk RA, Cerra FB, Dellinger RP, Fein AM, Knaus WA, Schein RM, Sibbald WJ. Definitions for sepsis and organ failure and guidelines for the use of innovative therapies in sepsis. The ACCP/SCCM Consensus Conference Committee. American College of Chest Physicians/Society of Critical Care Medicine. Chest. 1992;101:1644-55.

2. Pittet D, Rangel-Frausto S, Li N, Tarara D, Costigan M, Rempe L, Jebson P, Wenzel RP. Systemic inflammatory response syndrome, sepsis, severe sepsis and septic shock: incidence, morbidities and outcomes in surgical ICU patients. Intensive Care Med. 1995;21:302-9.

3. Menger MD, Vollmar B. Systemic inflammatory response syndrome (SIRS) and sepsis in surgical patients. Intensive Care Med. 1996;22:616-7.

4. Bone RC. Sepsis, sepsis syndrome, and the systemic inflammatory response syndrome (SIRS). Gulliver in Laputa. JAMA. $1995 ; 273: 155-6$

5. Haga Y, Beppu T, Doi K, Nozawa F, Mugita N, Ikei S, Ogawa M. Systemic inflammatory response syndrome and organ dysfunction following gastrointestinal surgery. Crit Care Med. 1997;25: 1994-2000.

6. Sibbald WJ, Doig G, Inman KJ. Sepsis, SIRS and infection. Intensive Care Med. 1995;21:299-301.

7. Baker SP, O'Neill B, Haddon W Jr, Long WB. The injury severity score: a method for describing patients with multiple injuries and evaluating emergency care. J Trauma. 1974;14:187-96.

8. Champion HR, Copes WS, Sacco WJ, Lawnick MM, Bain LW, Gann DS, Gennarelli T, Mackenzie E, Schwaitzberg S. A new characterization of injury severity. J Trauma. 1990;30:539-45.

9. Frith D, Brohi K. The acute coagulopathy of trauma shock: clinical relevance. Surgeon. 2010;8:159-63.

10. Lustenberger T, Turina M, Seifert B, Mica L, Keel M. The severity of injury and the extent of hemorrhagic shock predict the incidence of infectious complications in trauma patients. Eur $\mathbf{J}$ Trauma Emerg Surg. 2009;35:538-46.

11. Giannoudis PV. Current concepts of the inflammatory response after major trauma: an update. Injury. 2003;34:397-404.

12. Sauaia A, Moore FA, Moore EE, Lezotte DC. Early risk factors for postinjury multiple organ failure. World J Surg. 1996;20:392-400.

13. Knaus WA, Draper EA, Wagner DP, Zimmerman JE. APACHE II: a severity of disease classification system. Crit Care Med. 1985;13:818-29.

14. Brohi K, Singh J, Heron M, Coats T. Acute traumatic coagulopathy. J Trauma. 2003;54:1127-30.

15. MacLeod JB, Lynn M, McKenney MG, Cohn SM, Murtha M. Early coagulopathy predicts mortality in trauma. J Trauma. 2003;55:39-44. 
16. Maegele M, Lefering R, Yucel N, Tjardes T, Rixen D, Paffrath T, Simanski C, Neugebauer E, Bouillon B; AG Polytrauma of the German Trauma Society (DGU). Early coagulopathy in multiple injury: an analysis from the German trauma registry on 8724 patients. Injury. 2007;38:298-304.

17. Mica L, Albrecht K, Keel M, Trentz O. Independent predictors of early death of polytrauma patients: an analysis of 696 patients. J Trauma Treat. 2012;1:118. doi:10.4172/2167-1222.1000118.

18. Ertel W, Trentz O. Causes of shock in the severely traumatized patient: emergency treatment. In: Goris RJA, Trentz O, editors. The integrated approach to trauma care, the first 24 hours. Berlin: Springer; 1995. p. 78-87.

19. Trentz O, Friedl HP. Therapeutic sequences in the acute period in unstable patients. In: Goris RJA, Trentz O, editors. The integrated approach to trauma care, the first 24 hours. Berlin: Springer; 1995. p. 172-8.

20. Collicott PE, Hughes I. Training in advanced trauma life support. JAMA. 1980;243:1156-9.

21. Dümbgen L, Rufibach K. Maximum likelihood estimation of a log-concave density and its distribution function: basic properties and uniform consistency. Bernoulli. 2009;15:40-68.

22. R Development Core Team. R: a language and environment for statistical computing. Vienna: R Foundation for Statistical Computing. ISBN 3-900051-07-0, http://www.R-project.org. 2011.
23. Dümbgen L, Rufibach K. logcondens: computations related to univariate log-concave density estimation. J Stat Softw. 2011;39:1-28.

24. Bone RC, Balk RA, Cerra FB, Dellinger RP, Fein AM, Knaus WA, Schein RM, Sibbald WJ. American College of Chest Physicians/ Society of Critical Care Medicine Consensus Conference: definitions for sepsis and organ failure and guidelines for the use of innovative therapies in sepsis. Crit Care Med. 1992;20:864-974.

25. Asayama K, Aikawa N. Evaluation of systemic inflammatory response syndrome criteria as a predictor of mortality in emergency patients transported by ambulance. Keio J Med. 1998;47: $19-27$.

26. Miller PR, Munn DD, Meredith JW, Chang MC. Systemic inflammatory response syndrome in the trauma intensive care unit: who is infected? J Trauma. 1999;47:1004-8.

27. Bianchi ME. DAMPs, PAMPs and alarmins: all we need to know about danger. J Leukoc Biol. 2007;81:1-5.

28. Choudhry MA, Bland KI, Chaudry IH. Trauma and immune response-effect of gender differences. Injury. 2007;38:1382-91.

29. Guo RF, Ward PA. C5a, a therapeutic target in sepsis. Recent Pat Antiinfect Drug Discov. 2006;1:57-65. 